\section{Metamaterial spheroidal cavity to enhance dipole radiation}

Tadahiro Negishi, Danilo Erricolo, Piergiorgio L.E. Uslenghi

\begin{abstract}
Metamaterials have been considered for their potential to improve the radiation characteristics of sources. A novel geometry consisting of a semi-oblate spheroidal cavity containing two layers, one made of DPS and the other made of DNG metamaterial, and built underneath an aperture in infinite metallic plane is considered. An exact analytical solution is obtained and its numerical evaluation demonstrates a significant improvement of the directive radiation of a dipole source, with the appropriate combination of the DPS and DNG layers.
\end{abstract}

Index Terms-Artificial materials, Electromagnetic Radiation, Electromagnetic Scattering, Electromagnetic Analysis, Electromagnetic Fields, Electromagnetic Theory, Spheroids, Isorefractive Material

\section{INTRODUCTION}

The novelty of this article is that it focuses on the use of metamaterials to modify the distribution of the electromagnetic field due to a source, however its main difference compared to previous approaches [1]-[5] is that the source is not embedded in the metamaterial. In fact, our geometry consists of a dipole source immersed in an ordinary double positive (DPS) material with positive dielectric permittivity and positive magnetic permeability, illuminating a semi-oblate spheroidal cavity which is filled with a DNG metamaterial layer with negative dielectric permittivity and negative magnetic permeability, and with a layer made of the same DPS material outside the cavity. The cavity is built underneath an infinite metallic plane and connected to the outside through an aperture, as shown in the cross-section of Fig. 1.

An exact analytical solution for a dipole source located on the axis of symmetry and axially oriented is obtained in terms of infinite series containing oblate spheroidal functions. This geometry is relevant because it contains features such as sharp edges, an aperture, a cavity, and different materials. All these features make this new exact solution important as a canonical reference to validate the accuracy of numerical approaches, such as CAD software, to solve electromagnetic scattering problems.

The analytical results show that with a specific combination of the DPS and DNG layers inside the semi-oblate spheroidal cavity, the radiation of the dipole source is significantly more directive compared to the one obtained with related geometries such as those discussed by Berardi et al. in [6], Valentino and Erricolo [7], and Askarpour and Uslenghi in [8]. Preliminary results were presented in [9].

The time dependence $\exp (-i \omega t)$ is assumed and suppressed throughout.

Manuscript received date1; revised date2; accepted date3. Date of publication date4; date of current version date5

T. Negishi, D. Erricolo and P. Uslenghi are with the Department of Electrical and Computer Engineering, University of Illinois at Chicago, Chicago, IL, 60607, USA. (email:t.negishi@gmail.com; derric1@uic.edu; uslenghi@uic.edu). This research was partially supported by the US Air Force Research Laboratory with grant FA9550-12-1-0174.

\section{Geometry OF THE PROBlem}

The geometry of the problem has symmetry of rotation around the $\mathrm{z}$ axis and is shown in the cross section of Fig. 1. All surfaces are coordinate surfaces in the oblate spheroidal system, $(\eta, \xi, \varphi)$ related to the rectangular coordinates $(x, y, z)$ by $x=(d / 2) \sqrt{\xi^{2}+1} \sqrt{1-\eta^{2}} \cos \varphi, y=$ $(d / 2) \sqrt{\xi^{2}+1} \sqrt{1-\eta^{2}} \sin \varphi, z=(d / 2) \xi \eta$, where $\xi \geq 0$, $-1 \leq \eta \leq 1,0 \leq \varphi \leq 2 \pi$ and $d$ is the distance between the focal points $F_{1} \equiv(x=-d / 2, y=0, z=0) \equiv(\eta=$ $0, \xi=0, \varphi=\pi)$ and $F_{2} \equiv(x=d / 2, y=0, z=0) \equiv$ $(\eta=0, \xi=0, \varphi=0)$. Referring to Fig. 1 , there is a metallic plane at $z=0$ with a circular aperture of radius $d / 2$ that corresponds to the coordinate surface $\eta=0$. The half-space $z>0$ is filled by DPS material with dielectric permittivity $\varepsilon$ and magnetic permeability $\mu$. Below the aperture, there is a cavity limited by the metallic semi-oblate spheroidal surface at $\xi=\xi_{1}$. The interior of the cavity contains two regions. Region 1 is between the aperture $\xi=0$, the metallic plane $\eta=0$ and the oblate spheroidal surface $\xi=\xi_{2}$. Region 2 is between the oblate spheroidal surface at $\xi=\xi_{2}$, the plane $\eta=0$, and the metallic wall of the cavity along the oblate spheroidal surface $\xi=\xi_{1}$.

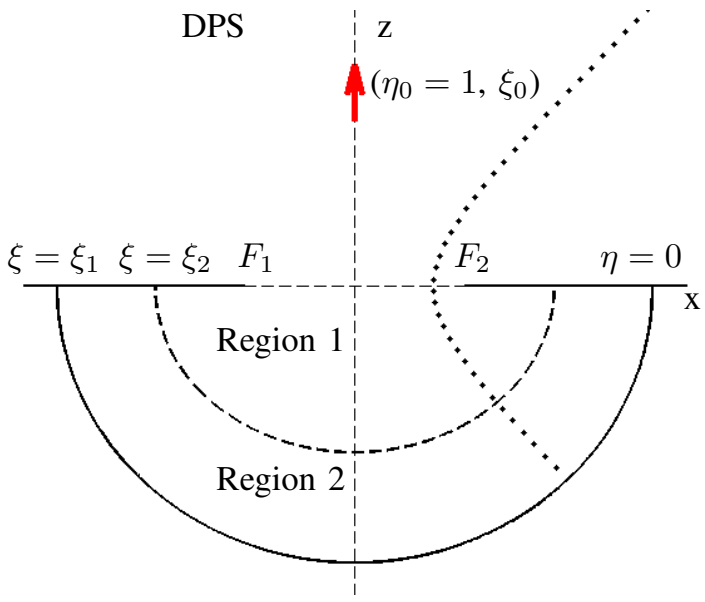

Fig. 1: Cross-section of the geometry of the problem. A dipole source located at $\left(\xi_{0}, \eta_{0}=1\right)$ illuminates the infinite metallic plane $\eta=0$ and the aperture $\xi=0$. The cavity has a diameter $d$ corresponding to the interfocal distance between $F_{1}$ and $F_{2}$. The dotted line represents a sample coordinate surface with $|\eta|=0.7$, a hyperboloid of revolution.

\section{ELECTRIC DIPOLE SOURCE}

For an electric dipole source located along the $\mathrm{z}$ axis above the ground plane at $\left(\xi_{0}>0, \eta_{0}=1\right)$ and axially oriented, the rotational symmetry of the problem requires that the field components be

$$
\begin{aligned}
\mathbf{H} & =H_{\varphi}(\eta, \xi) \hat{\varphi}, \quad H_{\eta}=H_{\xi}=0 \\
\mathbf{E} & =E_{\eta}(\eta, \xi) \hat{\eta}+E_{\xi}(\eta, \xi) \hat{\xi}, \quad E_{\varphi}=0
\end{aligned}
$$

where

$$
E_{\eta}=\frac{i Z}{c} \sqrt{\frac{\xi^{2}+1}{\xi^{2}+\eta^{2}}}\left(\frac{\partial}{\partial \xi}+\frac{\xi}{\xi^{2}+1}\right) H_{\varphi}
$$




$$
E_{\xi}=-\frac{i Z}{c} \sqrt{\frac{1-\eta^{2}}{\xi^{2}+\eta^{2}}}\left(\frac{\partial}{\partial \eta}-\frac{\eta}{1-\eta^{2}}\right) H_{\varphi}
$$

$Z$ is the wave impedance of the medium, and $c=k d / 2$ is the product of the wave number $k$ and the inter-focal radius $d / 2$. The constant $c$ is replaced by $-c$ in the DNG medium.

If the electric Hertz potential of the dipole is $\Pi_{e}=$ $\hat{\mathbf{z}} \exp (i k r) /(k r)$, where $r$ is the distance between the dipole $\left(\eta_{0}=1, \xi_{0}\right)$ and the field point $(\eta, \xi)$, then the corresponding magnetic field is [10]:

$$
\begin{aligned}
H_{\varphi}^{i}= & \frac{2 k^{2}}{Z_{1} \sqrt{\xi_{0}^{2}+1}} \sum_{n=1}^{\infty} \frac{(-i)^{n}}{\tilde{\rho}_{1, n} \tilde{N}_{1, n}} \times \\
& R_{1, n}^{(1)}\left(-i c, i \xi_{<}\right) R_{1, n}^{(3)}\left(-i c, i \xi_{>}\right) S_{1, n}(-i c, \eta) .
\end{aligned}
$$

where $R_{1, n}^{(1)}$ and $R_{1, n}^{(3)}$ are radial oblate spheroidal functions of the first and third kind, respectively, $S_{1, n}$ are angular oblate spheroidal functions, and $\tilde{\rho}_{1, n}$ and $\tilde{N}_{1, n}$ are normalization factors, according to the notation of Flammer [11]. The symbol $<(>)$ refers to the smaller (greater) between $\xi$ and $\xi_{0}$.

Outside the cavity, the total magnetic field may be written as the superposition of the incident field (3), the field reflected by the infinite metallic plane evaluated assuming that there is no aperture, and a diffracted field representing the perturbation due to the aperture and satisfying the radiation condition:

$$
\begin{aligned}
H_{\varphi}^{t} & =H_{\varphi}^{i}+H_{\varphi}^{r}+H_{\varphi}^{d}=\frac{-4 i k^{2}}{Z_{1} \sqrt{\xi_{0}^{2}+1}} \sum_{\ell=0}^{\infty} \frac{(-1)^{\ell}}{\widetilde{\rho}_{1,2 \ell+1} \widetilde{N}_{1,2 \ell+1}} \\
& \times S_{1,2 \ell+1}(-i c, \eta)\left[a_{\ell}^{(e)} R_{1,2 \ell+1}^{(3)}(-i c, i \xi)+\right. \\
& \left.R_{1,2 \ell+1}^{(1)}\left(-i c, i \xi_{<}\right) R_{1,2 \ell+1}^{(3)}\left(-i c, i \xi_{>}\right)\right] .
\end{aligned}
$$
is:

Inside the cavity, the total magnetic field in the DPS region

$$
\begin{aligned}
& H_{\varphi, \mathrm{DPS}}=\frac{-4 i k^{2}}{Z_{1} \sqrt{\xi_{0}^{2}+1}} \sum_{\ell=0}^{\infty} \frac{(-1)^{\ell}}{\widetilde{\rho}_{1,2 \ell+1} \widetilde{N}_{1,2 \ell+1}} S_{1,2 \ell+1}(-i c,-\eta) \\
& \times\left[b_{\ell}^{(e)} R_{1,2 \ell+1}^{(1)}(-i c, i \xi)+c_{\ell}^{(e)} R_{1,2 \ell+1}^{(3)}(-i c, i \xi)\right]
\end{aligned}
$$

whereas inside the DNG region is:

$$
\begin{aligned}
H_{\varphi, \mathrm{DNG}} & =\frac{-4 i k^{2}}{Z_{2} \sqrt{\xi_{0}^{2}+1}} \sum_{\ell=0}^{\infty} \frac{(-1)^{\ell}}{\widetilde{\rho}_{1,2 \ell+1} \widetilde{N}_{1,2 \ell+1}} S_{1,2 \ell+1}(i c,-\eta) \\
& \times\left[B_{\ell}^{(e)} R_{1,2 \ell+1}^{(1)}(i c, i \xi)+C_{l}^{(e)} R_{1,2 \ell+1}^{(3)}(i c, i \xi)\right] \quad(6)
\end{aligned}
$$

In the DPS region, $\varepsilon_{1}>0, \mu_{1}>0$ are the electric permittivity and magnetic permeability, respectively, $k_{1}=k=\omega \sqrt{\varepsilon_{1} \mu_{1}}>$ 0 the wavevector and $Z_{1}=\sqrt{\mu_{1} / \varepsilon_{1}}$ the material impedance. In the DNG region, $\varepsilon_{2}<0, \mu_{2}<0$ are the electric permittivity and magnetic permeability, respectively, $k_{2}=\omega \sqrt{\varepsilon_{2} \mu_{2}}<0$ the wavevector and $Z_{2}=\sqrt{\mu_{2} / \varepsilon_{2}}$ the material impedance. The DPS and DNG materials satisfy the anti-isorefractive condition, i.e. $k_{1}=-k_{2}$, while the impedances $Z_{1}$ and $Z_{2}$ are both positive, but different in general, hence we introduce the ratio $\zeta=Z_{1} / Z_{2}$.

The unknown modal coefficients $a_{\ell}^{(e)}, b_{\ell}^{(e)}, c_{\ell}^{(e)}, B_{\ell}^{(e)}$ and $C_{\ell}^{(e)}$ are found by imposing the boundary conditions, i.e. the continuity of the total tangential component of the magnetic and electric field across the aperture $\xi=0$, across the surface at $\xi_{2}$ and the vanishing of the electric field at the surface $\xi_{1}$. Note that in the application of the boundary conditions, the property that $S_{1,2 \ell+1}(-i c, \eta)=S_{1,2 \ell+1}( \pm i c,-\eta)$ and $S_{1,2 \ell}(-i c, \eta)=-S_{1,2 \ell}( \pm i c,-\eta)$ should be used.

If region 1 is DNG and region 2 is DPS, the modal coefficients are:

$$
\begin{aligned}
& a_{\ell}^{(e, m)}=\frac{t^{(e, m)}}{|M|}\left|\begin{array}{ll}
M_{2,2}^{(e, m)} & M_{2,3}^{(e, m)} \\
M_{3,2}^{(e, m)} & M_{3,3}^{(e, m)}
\end{array}\right| \\
& b_{\ell}^{(e, m)}=\frac{-t^{(e, m)}}{|M|}\left|\begin{array}{ll}
M_{2,1}^{(e, m)} & M_{2,3}^{(e, m)} \\
M_{3,1}^{(e, m)} & M_{3,3}^{(e, m)}
\end{array}\right| \\
& B_{\ell}^{(e, m)}=\frac{t^{(e, m)}}{|M|}\left|\begin{array}{ll}
M_{2,1}^{(e, m)} & M_{2,2}^{(e, m)} \\
M_{3,1}^{(e, m)} & M_{3,2}^{(e, m)}
\end{array}\right|,
\end{aligned}
$$

where $M_{m, n}^{(e, m)}$ is an element of the matrix

$$
\begin{aligned}
& M^{(e, m)}=\left(\begin{array}{lll}
M_{1,1}^{(e, m)} & M_{1,2}^{(e, m)} & M_{1,3}^{(e, m)} \\
M_{2,1}^{(e, m)} & M_{2,2}^{(e, m)} & M_{2,3}^{(e, m)} \\
M_{3,1}^{(e, m)} & M_{3,2}^{(e, m)} & M_{3,3}^{(e, m)}
\end{array}\right) \\
& M_{1,1}^{(e)}=R_{1,2 \ell+1}^{(3)}(-i c, 0)-\zeta R_{1,2 \ell+1}^{(3)}(i c, 0) \frac{R_{1,2 \ell+1}^{(3) \prime}(-i c, 0)}{R_{1,2 \ell+1}^{(3) \prime}(i c, 0)}
\end{aligned}
$$

$$
\begin{aligned}
M_{1,2}^{(e)} & =0, \quad M_{1,3}^{(e)}=-\zeta R_{1,2 \ell+1}^{(1)}(i c, 0) \\
M_{2,1}^{(e)} & =-\zeta R_{1,2 \ell+1}^{(3)}\left(i c, i \xi_{2}\right) \frac{R_{1,2 \ell+1}^{(3) \prime}(-i c, 0)}{R_{1,2 \ell+1}^{(3) \prime}(i c, 0)} \\
M_{2,2}^{(e)} & =R_{1,2 \ell+1}^{(1)}\left(-i c, i \xi_{2}\right) \\
& -R_{1,2 \ell+1}^{(3)}\left(-i c, i \xi_{2}\right) \frac{D_{2 \ell+1}^{(1)}\left(-i c, i \xi_{1}\right)}{D_{2 \ell+1}^{(3)}\left(-i c, i \xi_{1}\right)} \\
M_{2,3}^{(e)} & =-\zeta R_{1,2 \ell+1}^{(1)}\left(i c, i \xi_{2}\right) \\
M_{3,1}^{(e)} & =\frac{R_{1,2 \ell+1}^{(3) \prime}(-i c, 0)}{R_{1,2 \ell+1}^{(3) \prime}(i c, 0)} D_{2 \ell+1}^{(3)}\left(i c, i \xi_{2}\right) \\
M_{3,2}^{(e)} & =D_{2 \ell+1}^{(1)}\left(-i c, i \xi_{2}\right)-D_{2 \ell+1}^{(3)}\left(-i c, i \xi_{2}\right) \frac{D_{2 \ell+1}^{(1)}\left(-i c, i \xi_{1}\right)}{D_{2 \ell+1}^{(3)}\left(-i c, i \xi_{1}\right)}
\end{aligned}
$$

$M_{3,3}^{(e)}=D_{2 \ell+1}^{(1)}\left(i c, i \xi_{2}\right)$

$t^{(e)}=-R_{1,2 \ell+1}^{(1)}(-i c, 0) R_{1,2 \ell+1}^{(3)}\left(-i c, i \xi_{0}\right)$

$D_{n}^{(h)}( \pm i c, i \xi)=R_{1, n}^{(h) \prime}( \pm i c, i \xi)$

$$
+\frac{\xi}{\xi^{2}+1} R_{1, n}^{(h)}( \pm i c, i \xi), \quad(h=1,3)
$$

where the prime indicates partial derivative with respect to the argument $\xi$

$C_{l}^{(e)}=\frac{R_{1,2 \ell+1}^{(3) \prime}(-i c, 0)}{R_{1,2 \ell+1}^{(3) \prime}(i c, 0)} a_{l}^{(e)}, \quad c_{l}^{(e)}=-\frac{D_{2 \ell+1}^{(1)}\left(-i c, i \xi_{1}\right)}{D_{2 \ell+1}^{(3)}\left(-i c, i \xi_{1}\right)} b_{l}^{(e)}$

The far field behavior in the half space $z>0$ is obtained using the asymptotic expressions $c \xi \sim k r, \eta \sim \cos \theta$ as $c \xi \rightarrow \infty$, 
yielding

$$
\begin{aligned}
\left.H_{\varphi}^{t}\right|_{c \xi \rightarrow \infty} \sim & \frac{e^{i k r}}{k r} \frac{4 i k^{2}}{Z_{1} \sqrt{\xi_{0}^{2}+1}} \times \\
& \sum_{l=0}^{\infty} \frac{R_{1,2 \ell+1}^{(1)}+a_{l}^{(e)}}{\widetilde{\rho}_{1,2 \ell+1} \widetilde{N}_{1,2+1}} S_{1,2 \ell+1}(-i c, \cos \theta) .
\end{aligned}
$$

If region 1 is DPS and region 2 is DNG, the field expressions (4 - 6) and (22) in each region are still valid, however the modal coefficients change because the materials for regions 1 and 2 have been interchanged. The new modal coefficients are still formally expressed through eqs. (7-9) with superscript, where all symbols, except $t^{(e, m)}$, are now given by:

$$
\begin{aligned}
& \tilde{M}_{1,1}^{(e)}=2 R_{1,2 \ell+1}^{(3)}(-i c, 0) \\
& \tilde{M}_{1,2}^{(e)}=-R_{1,2 \ell+1}^{(1)}(-i c, 0) \\
& \tilde{M}_{1,3}^{(e)}=0, \quad \tilde{M}_{2,1}^{(e)}=R_{1,2 \ell+1}^{(3)}\left(-i c, i \xi_{2}\right) \\
& \tilde{M}_{2,2}^{(e)}=-R_{1,2 \ell+1}^{(1)}\left(-i c, i \xi_{2}\right) \\
& \tilde{M}_{2,3}^{(e)}=\zeta\left[R_{1,2 \ell+1}^{(1)}\left(i c, i \xi_{2}\right)-\frac{D_{2 \ell+1}^{(1)}\left(i c, i \xi_{1}\right)}{D_{2 \ell+1}^{(3)}\left(i c, i \xi_{1}\right)} R_{1,2 \ell+1}^{(3)}\left(i c, i \xi_{2}\right)\right] \\
& \tilde{M}_{3,1}^{(e)}= D_{2 \ell+1}^{(3)}\left(-i c, i \xi_{2}\right), \quad \tilde{M}_{3,2}^{(e)}=-D_{2 \ell+1}^{(1)}\left(-i c, i \xi_{2}\right) \\
& \tilde{M}_{3,3}^{(e)}=-D_{2 \ell+1}^{(1)}\left(i c, i \xi_{2}\right)+\frac{D_{2 \ell+1}^{(1)}\left(i c, i \xi_{1}\right)}{D_{2 \ell+1}^{(3)}\left(i c, i \xi_{1}\right)} D_{2 \ell+1}^{(3)}\left(i c, i \xi_{2}\right)(29) \\
& \tilde{C}_{l}^{(e)}=-\frac{D_{2 \ell+1}^{(1)}\left(i c, i \xi_{1}\right)}{D_{2 \ell+1}^{(3)}\left(i c, i \xi_{1}\right)} \tilde{B}_{l}^{(e)}, \quad \tilde{c}_{l}^{(e)}=-\tilde{a}_{l}^{(e)}
\end{aligned}
$$

\section{Magnetic Dipole SOURCE}

For a magnetic dipole source located above ground plane on the $\mathrm{z}$ axis at $\left(\eta_{0}=1, \xi_{0}\right)$ and axially oriented, the rotational symmetry of the problems requires that the field components be

$$
\begin{aligned}
\mathbf{E} & =E_{\varphi}(\eta, \xi) \hat{\varphi}, \quad E_{\eta}=E_{\xi}=0 \\
\mathbf{H} & =H_{\eta}(\eta, \xi) \hat{\eta}+H_{\xi}(\eta, \xi) \hat{\xi}, \quad H_{\varphi}=0
\end{aligned}
$$

where

$$
\begin{aligned}
& H_{\eta}=-\frac{i}{c Z} \sqrt{\frac{\xi^{2}+1}{\xi^{2}+\eta^{2}}}\left(\frac{\partial}{\partial \xi}+\frac{\xi}{\xi^{2}+1}\right) E_{\varphi} \\
& H_{\xi}=\frac{i}{c Z} \sqrt{\frac{1-\eta^{2}}{\xi^{2}+\eta^{2}}}\left(\frac{\partial}{\partial \eta}-\frac{\eta}{1-\eta^{2}}\right) E_{\varphi}
\end{aligned}
$$

If the magnetic Hertz potential of the dipole is $\Pi_{m}=$ $\hat{\mathbf{z}} \exp (i k r) /(k r)$ then the corresponding electric field is [10]:

$$
\begin{aligned}
E_{\varphi}^{i}= & -\frac{2 k^{2} Z_{1}}{\sqrt{\xi_{0}^{2}+1}} \sum_{n=1}^{\infty} \frac{(-i)^{n}}{\widetilde{\rho}_{1, n} \widetilde{N}_{1, n}} \times \\
& R_{1, n}^{(1)}\left(-i c, i \xi_{<}\right) R_{1, n}^{(3)}\left(-i c, i \xi_{>}\right) S_{1, n}(-i c, \eta) .
\end{aligned}
$$

Outside the cavity, the total electric field may be written as the superposition of the incident field, given by (33), the field reflected by the infinite metallic plane evaluated assuming that there is no aperture, and a diffracted field representing the perturbation due to the aperture.

$$
\begin{aligned}
E_{\varphi}^{t} & =E_{\varphi}^{i}+E_{\varphi}^{r}+E_{\varphi}^{d}=\frac{-4 i k^{2} Z_{1}}{\sqrt{\xi_{0}^{2}+1}} \sum_{\ell=1}^{\infty} \frac{(-1)^{\ell}}{\widetilde{\rho}_{1,2 \ell} \widetilde{N}_{1,2 \ell}} \\
& \times S_{1,2 \ell}(-i c, \eta)\left[a_{\ell}^{(m)} R_{1,2 \ell}^{(3)}(-i c, i \xi)\right. \\
& \left.+R_{1,2 \ell}^{(1)}\left(-i c, i \xi_{<}\right) R_{1,2 \ell}^{(3)}\left(-i c, i \xi_{>}\right)\right] .
\end{aligned}
$$

Inside the cavity, the total electric field in the DPS region is:

$$
\begin{array}{r}
E_{\varphi, \mathrm{DPS}}=\frac{-4 i k^{2} Z_{1}}{\sqrt{\xi_{0}^{2}+1}} \sum_{\ell=1}^{\infty} \frac{(-1)^{l}}{\widetilde{\rho}_{1,2 \ell} \widetilde{N}_{1,2 \ell}} S_{1,2 \ell}(-i c,-\eta) \times \\
{\left[b_{l}^{(m)} R_{1,2 \ell}^{(1)}(-i c, i \xi)+c_{l}^{(m)} R_{1,2 \ell}^{(3)}(-i c, i \xi)\right] .}
\end{array}
$$

whereas the total electric field inside the DNG region is:

$$
\begin{array}{r}
E_{\varphi, \mathrm{DNG}}=\frac{-4 i k^{2} Z_{2}}{\sqrt{\xi_{0}^{2}+1}} \sum_{\ell=1}^{\infty} \frac{(-1)^{l}}{\widetilde{\rho}_{1,2 \ell} \widetilde{N}_{1,2 \ell}} S_{1,2 \ell}(i c,-\eta) \times \\
{\left[B_{l}^{(m)} R_{1,2 \ell}^{(1)}(i c, i \xi)+C_{l}^{(m)} R_{1,2 \ell}^{(3)}(i c, i \xi)\right]}
\end{array}
$$

If region 1 is DNG and region 2 is DPS, the modal coefficients are given by eqs. (7-9) and matrix (10) with new matrix elements:

$$
\begin{aligned}
t^{(m)}= & -R_{1,2 \ell}^{(1) \prime}(-i c, 0) R_{1,2 \ell}^{(3)}\left(-i c, i \xi_{0}\right) \\
M_{1,1}^{(m)}= & R_{1,2 \ell}^{(3) \prime}(-i c, 0)-\zeta R_{1,2 \ell}^{(3) \prime}(i c, 0) \frac{R_{1,2 \ell}^{(3)}(-i c, 0)}{R_{1,2 \ell}^{(3)}(i c, 0)} \\
M_{1,2}^{(m)}= & 0, \quad M_{1,3}^{(m)}=R_{1,2 \ell}^{(1) \prime}(i c, 0) \\
M_{2,1}^{(m)}= & \zeta \frac{R_{1,2 \ell}^{(3)}(-i c, 0)}{R_{1,2 \ell}^{(3)}(i c, 0)} R_{1,2 \ell}^{(3)}\left(i c, i \xi_{2}\right) \\
M_{2,2}^{(m)}= & \zeta\left[R_{1,2 \ell}^{(1)}\left(-i c, i \xi_{2}\right)\right. \\
& \left.-\frac{R_{1,2 \ell}^{(1)}\left(-i c, i \xi_{1}\right)}{R_{1,2 \ell}^{(3)}\left(-i c, i \xi_{1}\right)} R_{1,2 \ell}^{(3)}\left(-i c, i \xi_{2}\right)\right] \\
M_{2,3}^{(m)}= & -R_{1,2 \ell}^{(1)}\left(i c, i \xi_{2}\right) \\
M_{3,1}^{(m)}= & -\zeta \frac{R_{1,2 \ell}^{(3)}(-i c, 0)}{R_{1,2 \ell}^{(3)}(i c, 0)} D_{2 \ell}^{(3)}\left(i c, i \xi_{2}\right) \\
M_{3,2}^{(m)}= & D_{2 \ell}^{(1)}\left(-i c, i \xi_{2}\right)-\frac{R_{1,2 \ell}^{(1)}\left(-i c, i \xi_{1}\right)}{R_{1,2 \ell}^{(3)}\left(-i c, i \xi_{1}\right)} D_{2 \ell}^{(3)}\left(-i c, i \xi_{2}\right) \\
c_{l}^{(m)}= & D_{2 \ell}^{(1)}\left(i c, i \xi_{2}\right) \\
C_{l}^{(m)}=-\frac{R_{1,2 \ell}^{(m)}\left(-i c, i \xi_{1}\right)}{R_{1,2 \ell}^{(3)}\left(-i c, i \xi_{1}\right)} b_{l}^{(m)} & \\
R_{1,2 \ell}^{(3)}(i c, 0) & R_{l}^{(m)}
\end{aligned}
$$

The far field behavior in the half space $z>0$ is obtained using the asymptotic expressions $c \xi \sim k r, \eta \sim \cos \theta$ as $c \xi \rightarrow$ 


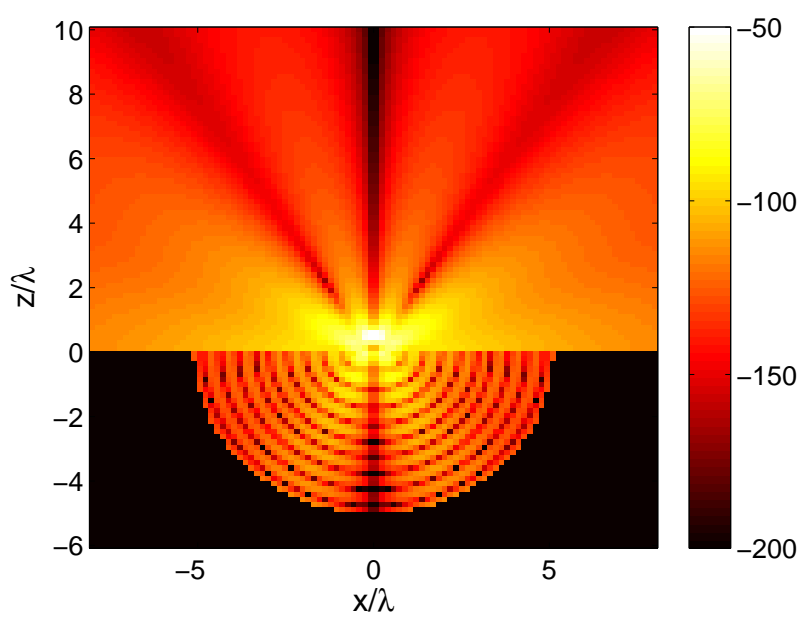

(a)

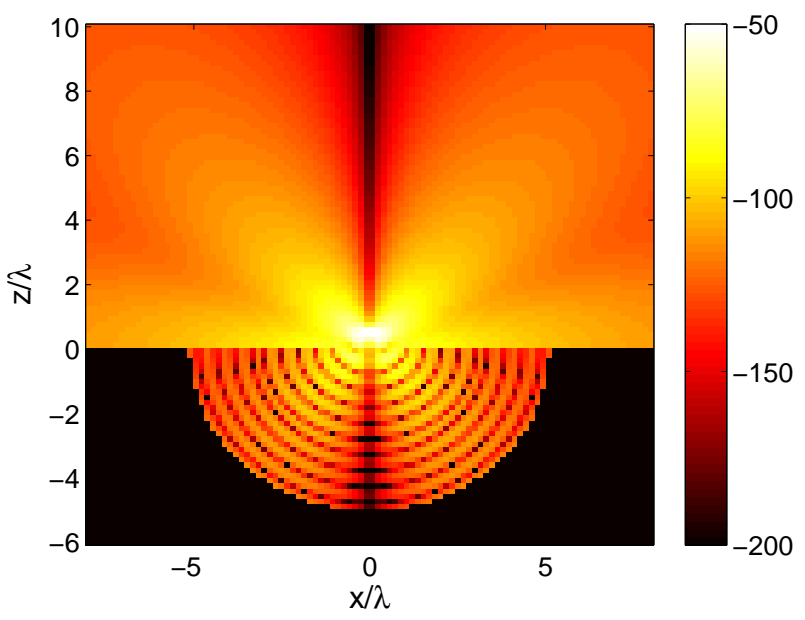

(b)

Fig. 2: Total magnetic field $\left|H_{\varphi}\right|$ due to an electric dipole located at $\left(z_{0} / \lambda=1 / 2, \xi_{0}=1 / 2\right)$ for an oblate spheroidal cavity with $c=2 \pi, d / \lambda=2, \xi_{1}=5, \xi_{2}=3, \zeta=0.5$. (a) Region 1 DNG and Region 2 DPS; and, (b) Region 1 DPS and Region 2 DNG.

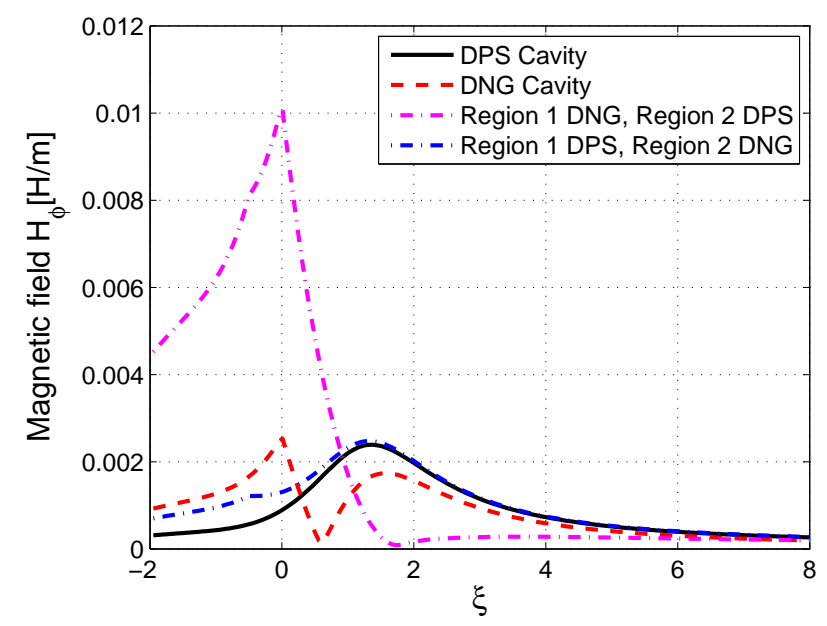

(a)

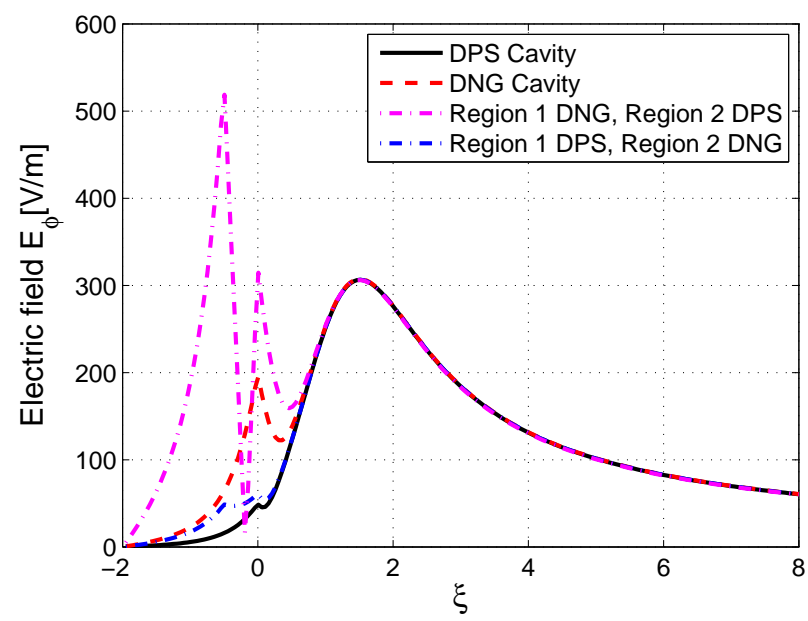

(b)

Fig. 3: (a) Total magnetic field $\left|H_{\varphi}\right|$ due to an electric dipole and (b) total electric field $\left|E_{\varphi}\right|$ due to magnetic dipole located at $\left(\xi_{0}=1.5, \eta_{0}=1\right)$ and evaluated along the coordinate line $|\eta|=0.7$ for a cavity with $c=1, d=\lambda / \pi, \zeta=0.5, \xi_{1}=2$ and $\xi_{2}=0.5$. Negative values of $\xi$ correspond to locations within the cavity. Also, the fields are evaluated with $\xi_{1}=\xi_{2}=2$ corresponding to an all DNG and an all DPS filled cavity.

$\infty$, yielding

$$
\begin{aligned}
\left.E_{\varphi}^{t}\right|_{c \xi \rightarrow \infty} \sim & \frac{e^{i k r}}{k r} \frac{4 i k^{2} Z_{1}}{\sqrt{\xi_{0}^{2}+1} \times} \\
& \sum_{\ell=1}^{\infty} \frac{R_{1,2 \ell}^{(1)}+a_{l}^{(m)}}{\widetilde{\rho}_{1,2 \ell} \widetilde{N}_{1,2 \ell}} S_{1,2 \ell+1}(-i c, \cos \theta) .
\end{aligned}
$$

If region 1 is DPS and region 2 is DNG, the field expressions (34 - 36) and (48) in each region are still valid, however the modal coefficients change because of different boundary conditions.

The new modal coefficients are still formally given by eqs. (7-9), where all symbols, except $t^{(e, m)}$, have changed and are given below:

$$
\begin{aligned}
& \tilde{M}_{1,1}^{(m)}=2 R_{2 \ell}^{(3) \prime}(-i c, 0), \tilde{M}_{1,2}^{(m)}=-R_{2 \ell}^{(1) \prime}(-i c, 0) \\
& \tilde{M}_{1,3}^{(m)}=0, \quad \tilde{M}_{2,1}^{(m)}=-\zeta R_{1,2 \ell}^{(3)}\left(-i c, i \xi_{2}\right) \\
& \tilde{M}_{2,2}^{(m)}=\zeta R_{1,2 \ell}^{(1)}\left(-i c, i \xi_{2}\right) \\
& \tilde{M}_{2,3}^{(m)}=-R_{1,2 \ell}^{(1)}\left(i c, i \xi_{2}\right)+\frac{R_{1,2 \ell}^{(1)}\left(i c, i \xi_{1}\right)}{R_{1,2 \ell}^{(3)}\left(i c, i \xi_{1}\right)} R_{1,2 \ell}^{(3)}\left(i c, i \xi_{2}\right)
\end{aligned}
$$




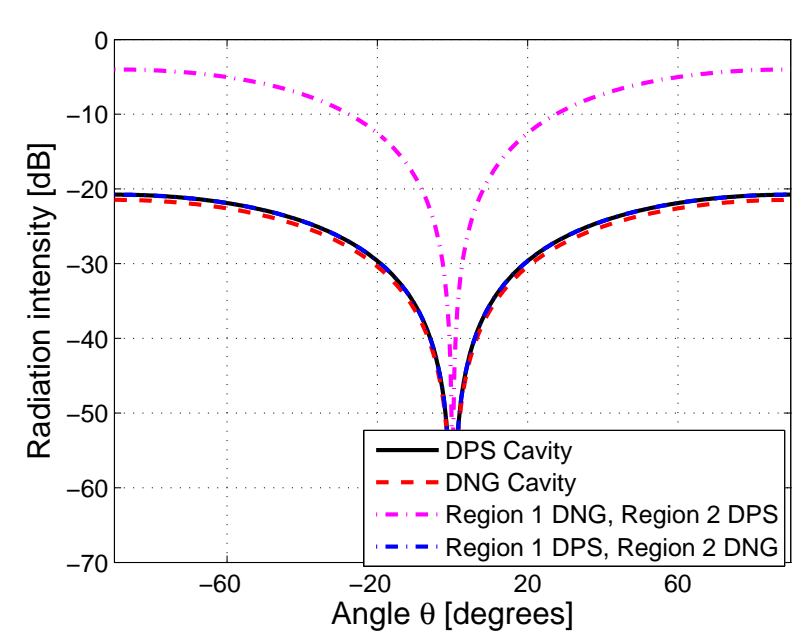

(a)

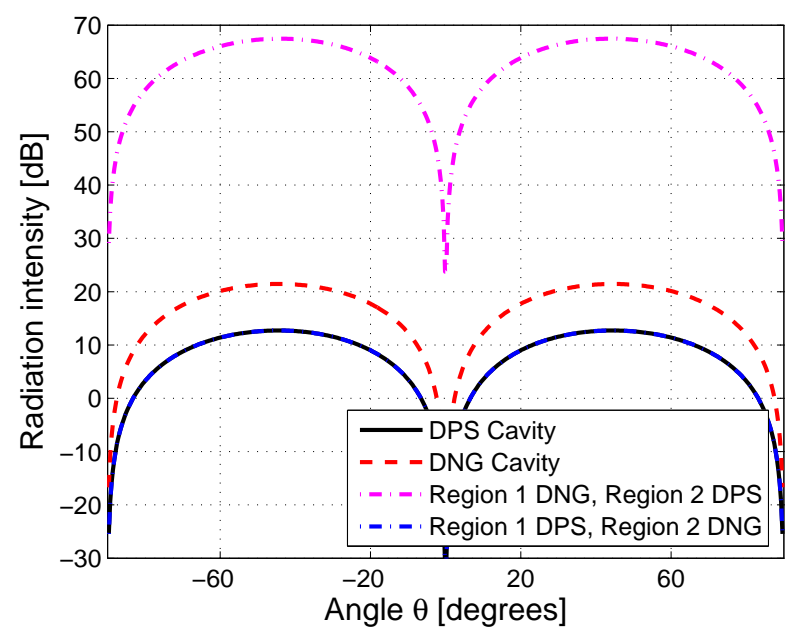

(b)

Fig. 4: (a) Radiation intensity $U^{(e)}$ due to an electric dipole and (b) radiation intensity $U^{(m)}$ due to a magnetic dipole located at $\left(z_{0} / \lambda=0.01, x_{0}=y_{0}=0\right)$ for $c=1, d / \lambda=0.3183, \zeta=0.5, \xi_{1}=1.205$ and $\xi_{2}=0.50$.

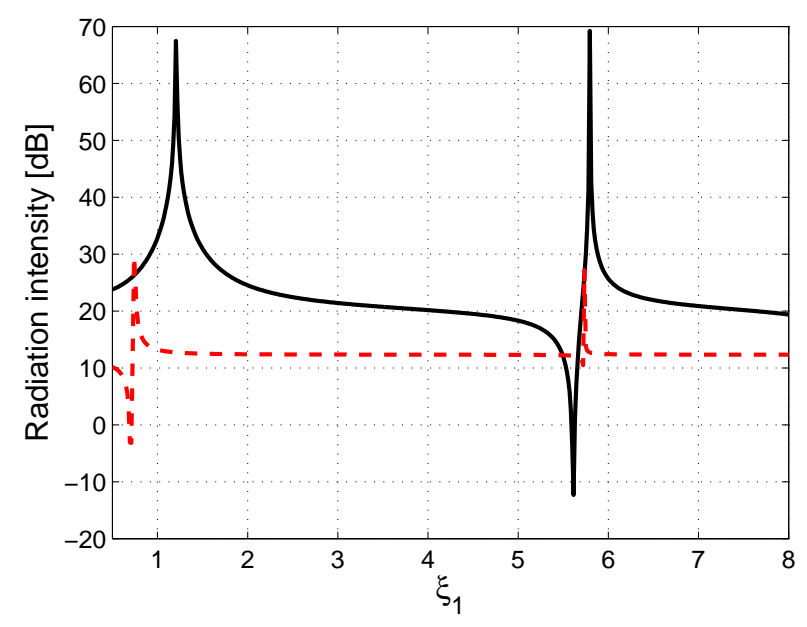

(a)

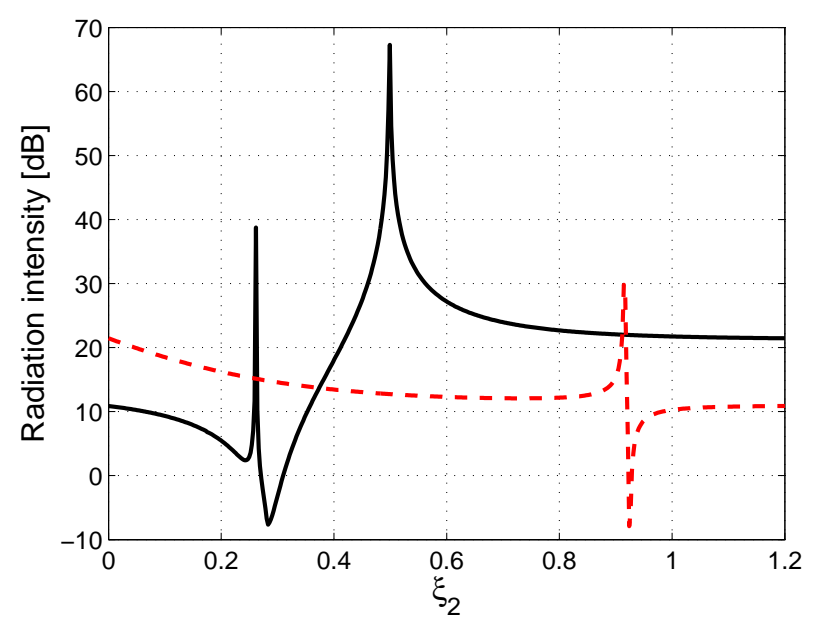

(b)

Fig. 5: Maximum radiation intensity $U^{(m)}\left(\theta_{\max }\right)$ due to a magnetic dipole located at $\left(z_{0} / \lambda=0.01, x_{0}=y_{0}=0\right)$ for $c=1$, $d / \lambda=0.3183, \zeta=0.5$, (a) $\xi_{2}=0.5$ and variable $\xi_{1}$ (b) $\xi_{1}=1.205$ and variable $\xi_{2}$. The solid black line refers to Region 1 DNG and Region 2 DPS, while the dashed red line refers to Region 1 DPS and Region 2 DNG.

$$
\begin{aligned}
& \tilde{M}_{3,1}^{(m)}=D_{2 \ell}^{(3)}\left(-i c, i \xi_{2}\right), \quad \tilde{M}_{3,2}^{(m)}=-D_{2 \ell}^{(1)}\left(-i c, i \xi_{2}\right) \\
& \tilde{M}_{3,3}^{(m)}=-D_{2 \ell}^{(1)}\left(i c, i \xi_{2}\right)+\frac{R_{1,2 \ell}^{(1)}\left(i c, i \xi_{1}\right)}{R_{1,2 \ell}^{(3)}\left(i c, i \xi_{1}\right)} D_{2 \ell}^{(3)}\left(i c, i \xi_{2}\right) \\
& \tilde{c}_{l}^{(m)}=-\tilde{a}_{l}^{(m)}, \quad \tilde{C}_{l}^{(m)}=-\frac{R_{1,2 \ell}^{(1)}\left(i c, i \xi_{1}\right)}{R_{1,2 \ell}^{(3)}\left(i c, i \xi_{1}\right)} \tilde{B}_{l}^{(m)}
\end{aligned}
$$

\section{NuMERICAL RESULTS}

Numerical results are provided only for the components of the magnetic and electric fields $H_{\varphi}, E_{\varphi}$ along the azimuth direction using eqs. (4-6) and (34-36), since all other polar- ization can be evaluated from these components using eqs. (2) and (32).

The criterion used to determine the convergence of the series was to keep on adding terms until their sum does not change more than $0.1 \%$. Accordingly it was sufficient to add the first 10 terms of each series.

Fig. 2 shows the total magnetic field $\left|H_{\varphi}\right|$ computed inside the cavity and outside the cavity near the aperture, when the source is an electric dipole and region 1 is DNG and region 2 is DPS and viceversa. Looking at these figures, one observes that there is a more complex field structure when Region 1 is DNG and Region 2 is DPS (Fig. 2a). This more complex behavior may be explained observing that there are more material variations going from the source towards the 
cavity. In fact, the material changes from DPS to DNG across the aperture and then from DNG to DPS across the boundary $\xi_{2}$. On the contrary, when Region 1 is DPS and Region 2 is DNG there is no change of material across the aperture and a transition from DPS to DNG across the boundary $\xi_{2}$.

Field magnitude values are better appreciated using the 1D plots of Fig. 3, which represents quantities evaluated along the coordinate line $|\eta|=0.7$ shown as dotted in Fig. 1. One should note that in Fig. 3 negative values of $\xi$ correspond to locations within the cavity. Fig. 3a shows the behavior of $\left|H_{\varphi}\right|$ for an electric dipole source. The same figure contains two additional results that help understanding the effect of the presence of metamaterial. The simplest situation corresponds to the whole cavity filled with DPS material, which is accomplished by using the exact expressions of this article by setting $\xi_{1}=$ $\xi_{2}=2$. This situation was already investigated in [6] and in fact our results match with Fig. 4 of [6] with $\zeta=1$. Then, the whole cavity filled with DNG metamaterial was investigated in [8] and our results reproduce the ones of Fig. 3(b) of [8] with $\zeta=0.5$. A more complex situation is considered in this manuscript, where the cavity contains two layered regions. It is apparent that the strongest value of the total magnetic field inside the cavity is achieved when Region 1 is DNG and Region 2 is DPS. Fig. 3b shows the behavior of $\left|E_{\varphi}\right|$ for the same geometry and materials already considered in Fig. 3a, but for the case of a magnetic dipole source. Similar to the results of Fig. 3a, it is apparent that the strongest field is achieved inside the cavity when Region 1 is DNG and Region 2 is DPS.

The third results are the radiation intensity plots which describe the behavior of the fields in the far-region and are given by

$$
\begin{aligned}
& U^{(e)}(\theta)=\frac{8 k^{2}}{Z_{1}\left(\xi_{0}^{2}+1\right)} \times \\
& \left.\mid \sum_{l=0}^{\infty} \frac{R_{1,2 \ell+1}^{(1)}\left(-i c, i \xi_{0}\right)+a_{l}^{(e)}}{\widetilde{\rho}_{1,2 \ell+1} \widetilde{N}_{1,2+1}} S_{1,2 \ell+1}(-i c, \cos \theta)\right)\left.\right|^{2}, \\
& U^{(m)}(\theta)=\frac{8 k^{2} Z_{1}}{\xi_{0}^{2}+1} \times \\
& \left|\sum_{\ell=1}^{\infty} \frac{R_{1,2 \ell}^{(1)}\left(-i c, i \xi_{0}\right)+a_{l}^{(m)}}{\widetilde{\rho}_{1,2 \ell} \widetilde{N}_{1,2 \ell}} S_{1,2 \ell}(-i c, \cos \theta)\right|^{2} .
\end{aligned}
$$

The radiation intensity for $d / \lambda=0.3183, \xi_{1}=1.205$ and $\xi_{2}=0.50$ is computed using eqs. (56-57), when the dipole sources are located very close to the ground plane $\left(z_{0} / \lambda=0.01, \xi_{0}=0.0628\right)$. Fig. 4 represents the results of the radiation intensity for the four cases studied in this article as well as with the limiting cases of the whole cavity filled with DPS and DNG material. The strongest peak occurs when Region 1 is DNG and Region 2 is DPS thus providing a more directive pattern. In other words, the layered combination of DNG and DPS produces a total field that is stronger than DNG and DPS standalone.

Finally, we show that with an appropriate combination of the dimensions of the DNG Region 1 and DPS Region 2 one could design an antenna that maximizes the radiation intensity. In fact, Fig. 4 suggests an investigation to determine if the larger values of radiation intensity already obtained for the combination of DNG for Region 1 and DPS for Region 2, could be further improved. Therefore, we seek to optimize the geometrical parameters $\xi_{1}$ and $\xi_{2}$ to maximize the radiation intensity $U\left(\theta_{\max }\right)^{(m)}$ in the case of a magnetic dipole source. In Fig. 5a, $\xi_{1}$ is varied and $\xi_{2}$ fixed, while in Fig. $5 \mathrm{~b} \xi_{2}$ is varied and $\xi_{1}$ fixed. One obtains that the radiation intensity is maximized when $\xi_{1}=1.205$ and $\xi_{2}=0.50$. In conclusion, a DNG metamaterial allows to obtain a maximum radiation intensity that significantly exceeds the one achievable with DPS material.

\section{CONCLUSION}

An exact analytical solution for axially located and oriented dipole with a two-layered semi-oblate spheroidal cavity partially filled with a metamaterial has been derived. The layered cavity augments the total field inside and outside the cavity. As a result, the radiation intensity has been increased compared to the cases with single-layered semi-oblate spheroidal cavities.

\section{ACKNOWLEDGEMENT}

This research was supported by the Air Force Office of Scientific Research of the U.S. Department of Defense through grant FA9550-12-1-0174. The authors would like to thank Dr. Amir Nader Askarpour for sharing useful Matlab codes.

\section{REFERENCES}

[1] R. W. Ziolkowski and A. Erentok, "Metamaterial-based efficient electrically small antennas," IEEE Trans. Antennas Propag., vol. 54, no. 7, pp. 2113-2130, July 2006.

[2] A. Alù, F. Bilotti, N. Engheta, and L. Vegni, "Metamaterial covers over a small aperture," IEEE Trans. Antennas Propag., vol. 54, no. 6, pp. 1632-1643, June 2006.

[3] H. Mirzaei and G. V. Eleftheriades, "A compact frequencyreconfigurable metamaterial-inspired antenna," IEEE Antennas Wirel. Propag. Lett., vol. 10, pp. 1154-1157, 2011.

[4] P.-Y. Chen, F. Monticone, and A. Alù, "Suppressing the electromagnetic scattering with an helical mantle cloak," IEEE Antennas Wirel. Propag. Lett., vol. 10, pp. 1598-1601, 2011.

[5] J. C. Soric, R. Fleury, A. Monti, A. Toscano, F. Bilotti, and A. Alù, "Controlling scattering and absorption with metamaterial covers," IEEE Trans. Antennas Propag., vol. 62, no. 8, pp. 4220-4229, Aug 2014.

[6] C. Berardi, D. Erricolo, and P. L. E. Uslenghi, "Exact dipole radiation for an oblate spheroidal cavity filled with isorefractive material and aperturecoupled to a half space," IEEE Trans. Antennas Propag., vol. 52, no. 9, pp. 2205-2213, Sept. 2004.

[7] M. Valentino and D. Erricolo, "Exact radiation of a dipole in the presence of a circular aperture in a ground plane backed by a spheroidal cavity and covered with an isorefractive diaphragm," Radio Sci., vol. 42, 2007, rS6S13, doi:10.1029/2006RS003548.

[8] A. N. Askarpour and P. L. E. Uslenghi, "Exact dipole radiation from an oblate semi-spheroidal cavity filled with DNG metamaterial," IEEE Trans. Antennas Propag., vol. 59, no. 7, pp. 2473-2479, July 2011.

[9] P.L.E.Uslenghi, D. Erricolo, and T. Negishi, "Radiation by a dipole antenna on the axis of a Semi-Spheroidal cavity partially filled with DNG metamaterial," in 2014 International Workshop on Antenna Technology (iWAT) (iWAT 2014), Sydney, Australia, Mar 2014.

[10] J. J. Bowman, T. B. A. Senior, and P. L. E. Uslenghi, Electromagnetic and Acoustic Scattering by Simple Shapes. New York: Hemisphere Publishing Corporation, 1987.

[11] C. Flammer, Spheroidal wave functions. Stanford University Press, 1957. 